\title{
Validating the efficacy of neurofeedback for optimising performance
}

John Gruzelier, Tobias Egner and David Vernon

\begin{abstract}
:
The field of neurofeedback training has largely proceeded without validation. Here we review our studies directed at validating SMR, beta and alpha-theta protocols for improving attention, memory, mood and music and dance performance in healthy participants. Important benefits were demonstrable with cognitive and neurophysiological measures which were predicted on the basis of regression models of learning. These are initial steps in providing a much needed scientific basis to neurofeedback, but much remains to be done.
\end{abstract}

\section{Introduction}

In this review of validation studies of EEG-, biofeedback (neurofeedback), the primary focus will be on the training of activity in the 12-14 $\mathrm{Hz}$ band, which is coincidental with the sensorimotor rhythm (SMR), and the adjacent beta. band $(15-20 \mathrm{~Hz})$. This focus is in recognition of Pfurtscheller's pioneering research in elucidating the behavioural significance of beta activity, SMR in particular, and putting it on the map in cognitive neuroscience (Pfurtscheller and Lopes da Silva, 1999).

In the EEG-neurofeedback field, the pioneering research on beta activity began with Sterman's operant conditioning studies with cats (see Sterman, 1996 for review). In cats during learned suppression of a bar press for food (the previously conditioned response), a particular brain rhythm emerged over the sensorimotor cortex with a frequency range of $12-20 \mathrm{~Hz}$ and with a spectral peak of $12-14 \mathrm{~Hz}$. The researchers successfully trained the cats to produce this 'SMR' through instrumental learning, by making a food reward contingent on the occurrence of SMR bursts (Wyrwicka and Sterman, 1968; Sterman et al., 1969). The associated behaviour was one of stillness, with SMR bursts regularly preceded 
by a drop in muscle tone. Subsequently, when the same cats participated in experiments to establish dose-response functions of a highly epileptogenic rocket fuel, they displayed elevated epileptic seizure thresholds compared with untrained cats. Importantly, the research was successfully extrapolated to humans, showing that epileptic motor seizure incidence could be lowered significantly by SMR feedback training (Sterman and Friar, 1972; Sterman et al., 1974; Sterman and MacDonald, 1978; Lantz and Sterman, 1988; Sterman, 2000).

This apparent quieting effect of SMR training on the excitability of the sensorimotor system inspired Lubar and co-workers to apply a protocol of SMR enhancement to the treatment of attention deficit hyperactivity disorder (ADHD) (Lubar and Shouse, 1976). In the ADHD field, this SMR protocol has often been used with the one that trains increments in higher components, such as the beta1 band (15-18 Hz), along with suppression of theta activity. Beta activity has often been associated with states of high alertness, concentration and focused attention (e.g. Gomez et al., 1998; Vazquez Marrufo et al., 2001; Kristeva-Feige et al., 2002). The low levels of beta produced by children with ADHD (Clarke et al., 1998, 2001; Monastra et al., 1999) are thought to have a detrimental effect on their ability to focus and concentrate. Training beta activity may also benefit those children suffering predominantly from problems of inattention and/or low arousal and not hyperactivity.

It was cogently hypothesised (Lubar and Shouse, 1976; Shouse and Lubar, 1979; Lubar and Lubar, 1984) that voluntary production of the SMR in the ADHD child would reduce hyperactive/impulsive behaviours whilst simultaneously improving attentional capabilities. Attentional abilities may also be enhanced by training low beta activity. To date, there have only been a handful of controlled studies demonstrating a variety of beneficial effects for ADHD (Linden et al., 1996; Thompson and Thompson, 1998; Monastra et al., 2001; Fuchs et al., 2003, and see for review Monastra et al., 2005). Because of a lack of large-scale randomised controlled studies, insufficient evidence exists at this time to support conclusively the effectiveness of EEG biofeedback for ADHD children. Notwithstanding, the studies to date have provided more than suggestive 
evidence for neurofeedback's potential for enhancing attention in clinical groups, and as we will now document, for improving attentional abilities in healthy people. Furthermore, evidence of validation of neurofeedback for enhancing a range of psychological processes is beginning to emerge.

\section{Validation of the influence of neurofeedback training on attention in healthy participants}

Most previous studies that have attempted to portray any association between EEG parameters and behavioural-dependent measures have done so by merely documenting some kind of post-training EEG changes that were presumably related to the actual training process, and further presumably related to behavioural performance changes (e.g. Tansey and Bruner, 1983; Lubar and Lubar, 1984; Tansey, 1993; Monastra et al., 2001;). However, in none of these studies have the corresponding correlation statistics been reported. Although attempts to formulate learning success on the basis of in-training EEG measures have been made in order to classify trainee participants as learners or nonlearners (Shouse and Lubar, 1979; Lubar et al., 1995; Rasey et al., 1996), these stopped short of directly correlating learning indices with changes in outcome measures, be they behavioural or electrophysiological. Arguably, the earliest case study reports by Lubar and Shouse (1976) came closest to specifically documenting the purported link between fulfilling feedback learning criteria and changes in dependent measures, but their results were of a descriptive nature and the implications were further hampered by a small number of subjects.

Accordingly, studies with ADHD children, though important, had not established a direct association between the ability to learn to enhance the desired frequency band in the EEG and the improvement in behaviour and cognition. Egner and Gruzelier set out to explore whether similar cognitive improvements could be achieved through training with the neurofeedback protocols, and whether improvements in attention could be predicted on the basis of regression models of indices of learning ability to increase relative and absolute SMR and beta1 amplitudes within each session. This would provide necessary validation of the 
effects on attention of these training protocols, and support the assumption that enhancing SMR over sensorimotor cortex will reduce the impulsive behaviour characteristic of ADHD, and the assumption that enhancing beta1 activity will improve sustained attention.

In the first study (Egner and Gruzelier, 2001), conservatoire students were trained on an attention-targeting $\mathrm{SMR}(\mathrm{C} 4) /$ beta1(C3) neurofeedback protocol involving ten 15-min sessions of both SMR and beta training. The assessment of attention was carried out employing a computerised continuous performance test (CPT) displaying two classes of stimuli: "targets" which required the participant to respond as quickly and accurately as possible by pushing a response switch, and "nontargets" which required the participant to refrain from responding. This task has been widely used in ADHD research (Monastra et al., 2005). Two types of errors can be made on such a task: errors of omission by failing to respond to a target stimulus, and errors of commission by erroneously responding to a nontarget stimulus. These errors are held to reflect inattentiveness and impulsiveness, respectively. A further attention measure has been derived from signal detection theory (Green and Swets, 1966), and termed "perceptual sensitivity" or "d prime" $\left(d^{0}\right)$. This takes into account both error types by expressing a ratio of hit rate to false alarm rate.

From the emergent data, it was established that ten training sessions of both SMR and beta neurofeedback led to a significant reduction in commission errors as compared with measures taken prior to training. When exploring the link between the process of learned EEG self-regulation and the reduction in impulsive mistakes, it was found that the relative success at enhancing the SMR was highly positively correlated with reduced commission errors. This means that participants who did well on the SMR feedback task were the ones who most reduced their impulsive mistakes after training. These findings support the notion that learned SMR enhancement is associated with improved response inhibition, and they constitute the first evidence for cognitive performance enhancement through neurofeedback in healthy volunteers. The results are shown in Fig. 1. 
The attention-enhancing potential of beta1 neurofeedback was also corroborated by electrocortical performance measures related to selective attention processes, results shown in Fig. 2. Beta1 training was associated with increments in the P300b event-related brain potential on a task that required active monitoring and detection of auditory target stimuli. Specifically responses to target stimuli increased significantly at frontal, central and parietal locations. The P300b has been conceptualised as representing activity in neuronal sources responsible for updating relevant stimulus environment information in working memory (Donchin and Coles, 1988). Of critical theoretical importance was the finding that both SMR and beta learning correlated positively with the P300b increases.

In a second study (Egner and Gruzelier, 2004), the main results were replicated using separate groups of conservatoire students for SMR and beta1 training and compared with a control group. Effects were again measured with a CPT and also a more complex divided attention task. Here stronger effects were demonstrated with the more complex divided attention task. SMR training benefited omission errors and reduced reaction time variability, while the $d^{0}$ measure improved on both tasks. Beta1 training was followed by reduced reaction times on the less complex CPT, and as in the earlier study there were larger P300b amplitudes, especially at central and parietal placements and in keeping with the posterior scalp distribution of the P300b, as before.
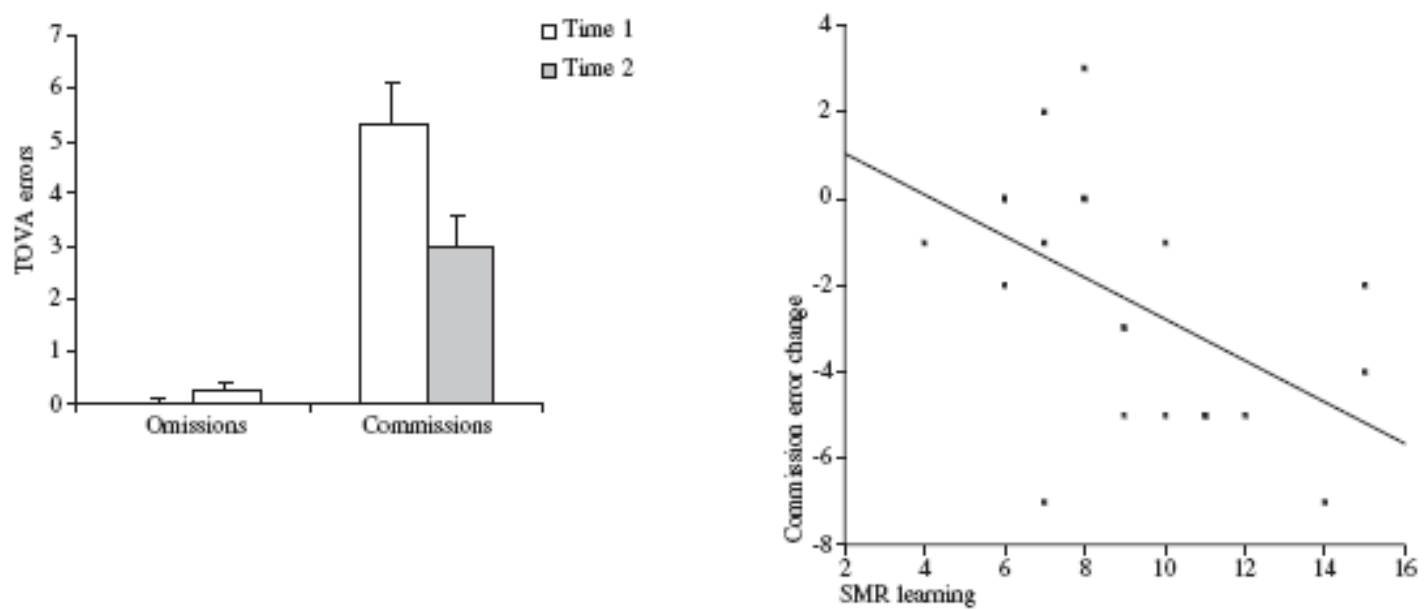

Fig. 1. Pre-to post-training change scores for errors of omission and commission (top panel) and regression line of best fit for the correlation between SMR learning index and commission error reduction (bottom panel). 
In a third study (Vernon et al., 2003), students were randomised to one of three groups: SMR training while inhibiting theta and beta $(18-22 \mathrm{~Hz})$, eyes open theta training while inhibiting delta and alpha, and a non-training control group. There were eight sessions with a $\mathrm{Cz}$ electrode placement. The effect of training was compared on a CPT with a two-or three-digit sequence target, which varied the memory load, and on a semantic working memory task with words presented in semantic clusters or randomly. There was clear evidence of operant control over the SMR, but participants were unable to achieve this with eyes open theta training. In the two-digit CPT with SMR training, there were highly significant reductions in both errors of omission and commission, which were not observed in the other groups, results shown in Fig. 3. But there was no advantage to SMR training with the three-digit task. More robust effects were found with the semantic working memory task in favour of SMR training. As shown in Fig. 4, improvements of the order of $10 \%$ were found with both clustered and unclustered recall with only eight sessions of training.

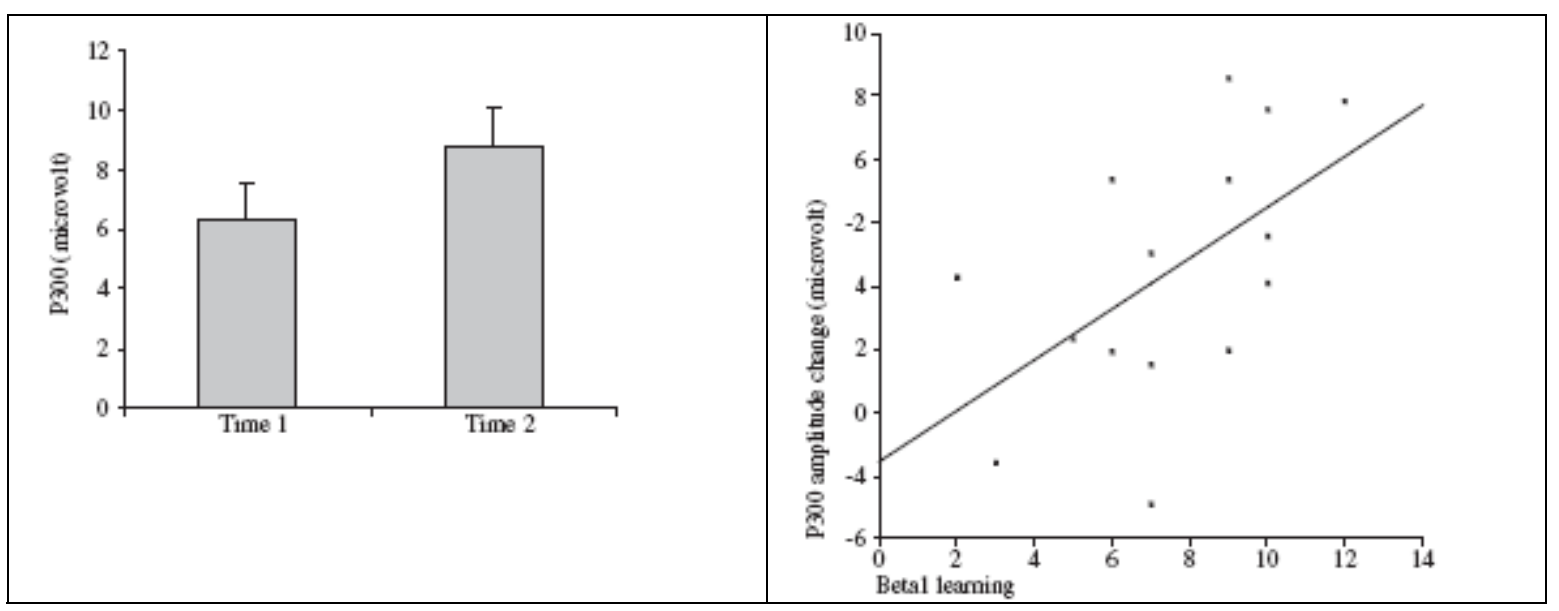

Fig. 2. Pre-to post-training change scores for P300 ERP mean amplitudes (top panel) and regression line of best fit for the correlation between beta1 learning index and P300 increments (bottom panel).

Together the results of these three experiments have significance for the treatment of ADHD. There was some evidence in all three experiments that impulsive errors on CPT tasks may be reduced following SMR training. Omission errors also benefited, along with an increase in perceptual sensitivity $\left(d^{\circ}\right)$. 
Furthermore, improvements in attention in conservatoire students could be predicted on the basis of regression models of indices of learning ability to increase relative and absolute SMR and beta1 amplitudes within each session. In addition, the increments in SMR and beta1 activity could predict the increases in P300b amplitude. This validation of the effects on attention of these training protocols widely used with ADHD children had not been previously demonstrated. The results support the assumption that enhancing SMR over sensorimotor cortex will reduce the impulsive behaviour characteristic of ADHD, and together with beta1 training will improve focused attention.

We have an ongoing controlled study comparing SMR and beta training in children with ADHD who were randomly assigned to neurofeedback or to a computerised attention test. A preliminary analysis of the first 16 participants on measures of attention has provided suggestive results favouring neurofeedback (Batty et al., 2005). The neurofeedback schedule consisted of 15 sessions of SMR training followed by 15 sessions of beta training, both with suppression of theta activity. At the time of the preliminary analysis, seven children had received neurofeedback and nine attention training. Advantages for neurofeedback over attention training were found with the dependent variables of errors of omission on the same CPT task used with students, and accuracy on an attention network test. The latter result was interpreted as indexing improved focussing attention through the processes of orienting and the selection of information. 

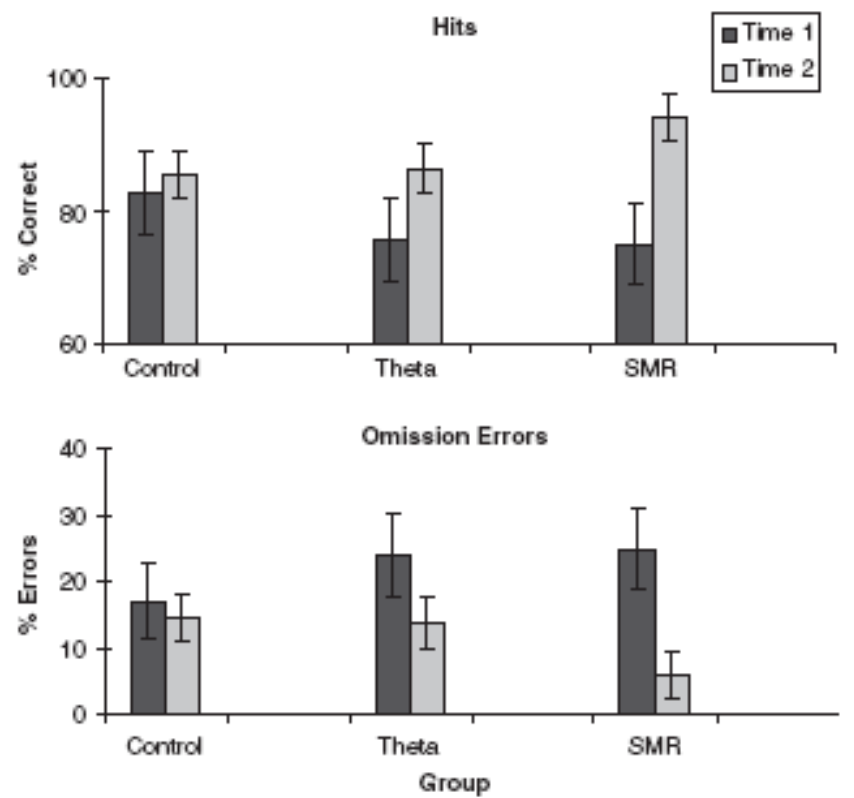

Fig. 3. Percentage hits (top panel) and omission errors (bottom panel) for 2-sequence attention CPT at time 1 and time 2 for the control, theta and SMR groups.

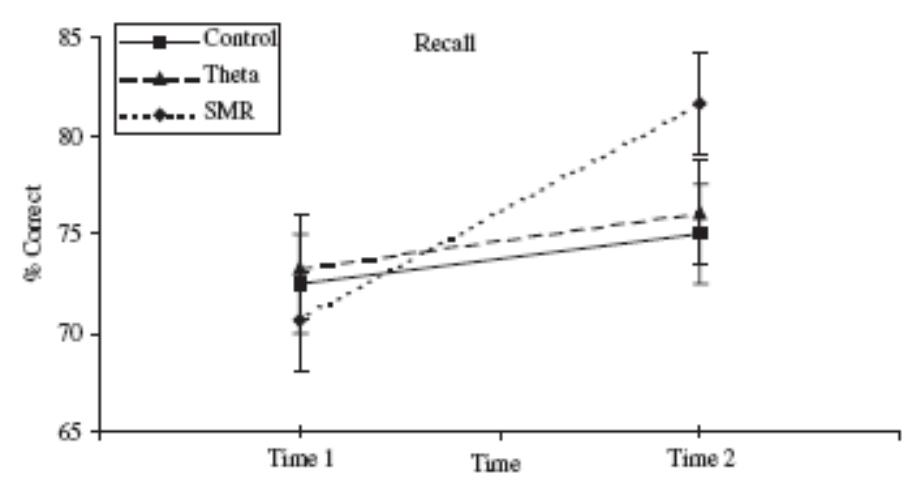

Fig. 4. Percentage correct recall (with standard error bars) for semantic working memory task, collapsed across non-clustered and clustered versions, at time 1 and time 2 for the control, theta and SMR groups.

Most recently Hanslmayr et al. (2005) examined in normal volunteers the efficacy on mental rotation performance of training a $2 \mathrm{~Hz}$ upper alpha band, which overlaps with the "SMR" band, and on a different trial inhibiting a $2 \mathrm{~Hz}$ theta band. Bands were individually adjusted according to the EEG spectrum recorded in a resting state with eyes closed. Subjects were classified into two groups: nine

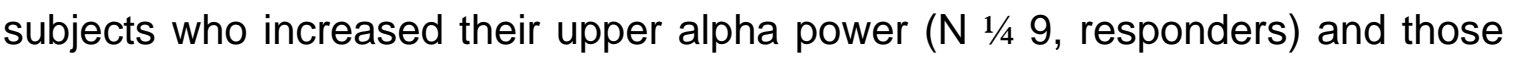
who decreased it ( $\mathrm{N}^{1 / 4} 10$, non-responders). Only one training session was given. Responders improved in their mental rotation performance, and a positive correlation was found between the degree of alpha enhancement and the improvement in mental rotation performance. Furthermore, in the trial intervals 
preceding mental rotation, they showed an increase in upper alpha power in keeping with relations between upper alpha activity and cognition (Klimesch et al., 2003).

Before considering further the implications of these results, our work in validating a slow wave training protocol and its effects on performance will be briefly outlined, not the least because comparisons were made with SMR and beta training.

\section{Validation of alpha-theta training}

This involves increasing the ratio between theta $(4-8 \mathrm{~Hz})$ and the usually more dominant alpha activity $(8-12 \mathrm{~Hz})$ in a state of eyes-closed relaxation. It is a widely used clinical and peak performance protocol. Origins lie with the first ever application of neurofeedback training by Kamiya (1962), which involved alpha training. This was reported to be relaxing and peaceful, and while initial attempts met with mixed success (Brown, 1970; Budzynski and Stoyva, 1972; Lynch et al., 1974; Pressner and Savitsky, 1977; Hardt and Kamiya, 1978; Plotkin and Rice, 1981), subsequently an alpha/theta neurofeedback protocol producing an hypnogogic state was developed to alleviate anxiety associated with alcoholism (Peniston and Kulkosky, 1989, 1990; Saxby and Peniston, 1995), and posttraumatic stress disorder (PTSD; Peniston and Kulkosky, 1991). This protocol has recently been successfully used with crack and cocaine misuse (Scott et al, 2005). We conducted a series of studies to establish the necessary evidence of operant control over the alpha/theta ratio, to establish its ecological validity for performance enhancement and benefits for well-being. Additional implications for this review include comparisons made with SMR and beta training.

In the first study, conservatoire students were randomly assigned to a mixed course of beta1/ SMR and alpha/theta training, to a no-training control group, or to the neurofeedback protocols combined with mental skills training and aerobics (Gruzelier et al., 2002; Egner and Gruzelier, 2003). Improvements in performance were found in the neurofeedback-only group, but not in the 
neurofeedback group engaging in additional interventions, nor in the no-training control group. The neurofeedback group improved most markedly on ratings of overall quality of performance and of their musicality and creativity.

Importantly, it was an alpha/theta training learning index, reflecting increasing ease at raising theta over alpha activity across the training process that correlated highly positively with music performance improvements. The SMR and beta protocols were unrelated. The benefits could not be attributed to a reduction in pre-performance state anxiety, for anxiety was successfully reduced in all groups. These findings supplied evidence for a potential benefit of training the alpha-theta ratio on a highly ecologically valid music performance measure.

In a constructive replication participants were randomly allocated to one of six groups: an alpha/ theta, SMR or beta1 neurofeedback training group, a physical exercise program, a mental skills training program or the Alexander technique, an established tool for improving performance in music conservatories worldwide. Music performance was assessed by ratings from expert judges blind to the experimental conditions, as before, and confirmed that it was the alpha/theta group that displayed significant improvements. Neither the SMR nor the beta group exhibited any post-training performance changes. Similarly, students from the Alexander technique, physical exercise and mental skills training groups showed no post-training changes. The results are shown in Fig. 5. Thus, the alpha/theta protocol's performance-enhancing effects proved to be replicable, particularly with respect to parameters on the "musicality" and other artistic evaluation categories including interpretative imagination, i.e. imagination in performance in interpretation of the score, in other words creativity. Thus, the alpha/theta training led to improvements on attributes of creativity and artistic expression as opposed to technical skills. It is noteworthy that individual participants displayed improvements of over $50 \%$ on some evaluation criteria while the average improvements were equivalent to two academic grades within the conservatory assessment system. The effects clearly were of professional significance. 


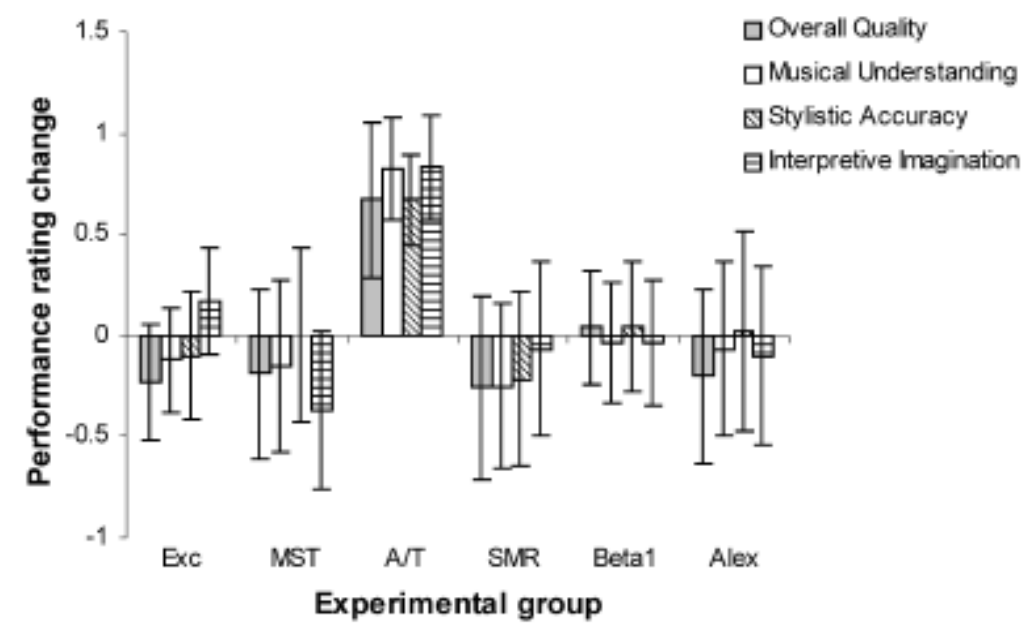

Fig. 5. Neurofeedback and music performance. Mean change scores (7SEM) for the physical exercise (Exc), mental skills training (MST), alpha/theta (A/T), SMR (SMR), beta1 (Beta1) and Alexander technique (Alex) groups on a 10-point rating scale of musical evaluation criteria. The a/t group displays musical improvements in overall quality $(+14.4 \%)$, musical understanding $(+16.4 \%)$, stylistic accuracy $(+13.5 \%)$ and interpretative imagination $(+17 \%)$.

These results suggest that alpha/theta training appears reliably to enhance artistic aspects of musical performance skills, independently of training on SMR and beta protocols, and that these effects are superior to the other interventions in this respect. As in the first study, all groups reported significantly less preperformance anxiety prior to the post-training performance, with no differences between groups, so that the benefits could not be attributed to stress reduction. Furthermore, the fact that music performance quality changes were not related to SMR and beta1 learning suggests that improvements were not mediated by attention-related variables.

Subsequently, the performance-enhancing effects were extended to competitive dance performance with a university ballroom and Latin dance team (Raymond

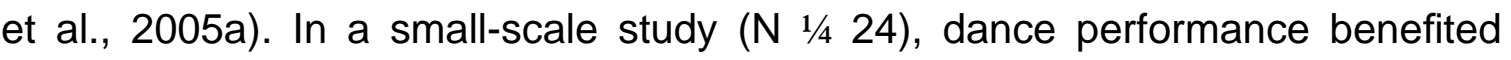
overall, and in particular timing. Interestingly, dance performance also benefited from heart rate variability coherence training with particular influence on dance technique. Again the benefits were of professional significance and occurred in only 5 weeks of training, while a non-intervention control group did not improve over this period, although their practice diaries disclosed that they practiced more. 
In order to explore possible benefits for wellbeing socially anxious and withdrawn students were randomised to alpha/theta training or to mock training where feedback was non-contingent (Raymond et al., 2005b), a strategy used previously in Egner et al. (2002). Advantages for the contingent feedback group were reported on self-ratings of composure, agreeableness, elevated mood, confidence and energy. There was a $25 \%$ improvement in mood overall, whereas the control non-contingent feedback group showed improvements in composure but coupled with less energy.

\section{Implications of validation for training}

With SMR, beta1 and alpha/theta training protocols, the principle strategy was accomplished of revealing a possible causal link between neurofeedback training and dependent measure changes. Considering first SMR training, both behavioural and neurophysiological changes were related to an index that reflected relative success at satisfying the operant feedback contingencies directly. But a more critical achievement was that learning indices were capable of predicting both the cognitive improvements in attention and the neurophysiological enhancement of the $\mathrm{P} 300 \mathrm{~b}$ response. SMR training did disclose benefits for both impulsive and inattentive aspects of attention performance. In fact when the two aspects were combined in the $\mathrm{d}^{0}$ metric, perceptual sensitivity was enhanced following SMR training in both studies and across visual and auditory sensory modalities. In sum, both formulation and assessment of meaningful neurofeedback performance-based predictors of the dependent measures were demonstrated.

In terms of the neurophysiological processes underlying the improvements in attention, our studies provide support for Sterman's (1996) proposal that there is decreased somatosensory and motor interference in cognitive processing as a result of SMR training, such that in ADHD it is the hyperactivity that disrupts attention, learning and memory. Thus, the putative improved regulation of sensorimotor/somatosensory pathways reduces processing interference from irrelevant stimuli and so facilitates the cognitive integration of the task- relevant 
stimuli. This was demonstrated in both the behavioural and neurophysiological tasks. For a more recent review of the neurophysiology see Sterman (2000).

Turning to beta1 training, in keeping with traditional notions of its association with generic cortical activation, and hence its application to cortically under-aroused ADHD children and adults, commission error reduction was negatively correlated with learning (Egner and Gruzelier, 2003), shorter reaction times (Egner and Gruzelier, 2004) and in both reports increased P300b amplitudes. These combined effects are compatible with an increase in the background cortical arousal in keeping with the proposal of raising cortical excitation in underaroused AD/HD children.

With alpha/theta training again correlations were found between learning indices and degree of performance improvement. We have provided the necessary evidence for operant control of the alpha/theta ratio, and the first evidence of the efficacy of the alpha-theta protocol as a sole intervention. An explanation for the alpha-theta effects based on generic relaxation can be discounted on the grounds that alpha/theta training was not associated with a greater decrease in pre-performance anxiety than that seen in other groups (see also Egner et al., 2002). In considering how alpha/ theta training achieves these remarkable effects, it has been proposed that the advantages for the slower rhythms in longdistance connectivity in the brain facilitates memory associations together with sensory-motor integration (Gruzelier and Egner, 2004; Gruzelier, 2006).

Along with the outcome of SMR and beta1 training, the alpha/theta effects cannot be accounted for by invoking practice, motivational or generic neurofeedback factors. Germaine to this were the opposite effects on attention that were found with SMR and beta1 training. SMR enhancement had the positive effects and beta1 enhancements the negative effects on impulsive response tendencies. This provides evidence of protocol specificity. The demonstration of protocol specificity also counters scepticism about neurofeedback being attributable to non-specific factors such as therapist contact or motivation. It also questions unitary theories of neurofeedback efficacy based on thalamocortical regulation and positing interchangeability of protocols. In other words, the notion that all that 
is important in the learning of self-regulation of the EEG is the bandwidth chosen is arbitrary and is not supported by our findings that SMR and beta1 training had opposite effects on impulsivity.

But most importantly our validation places SMR and beta1 training on a firmer footing in applications to ADHD in children and adults, while the improvement in semantic memory have implications for neurorehabilitation and addressing the ageing process in the elderly. The outcome of the alpha/theta training effects with music students confirmed a significantly beneficial effect on a highly ecologically valid and pedagogically relevant performance measure, while the size of the performance improvements in music and dance implies great potential for the implementation of this application in the performing arts, and studies are underway to extend the applications for music and dance.

It is perhaps remarkable that the benefits with neurofeedback training were achieved by only 10 sessions of training, and certainly clinical samples will require longer training. The next steps in the validation process will be to duplicate these studies in clinical groups and in the case of AD/HD in the inattentive, hyperactive and combined subtypes, as in our ongoing study (Batty et al., 2005). Can this efficacy be measured not only by clinical outcome but also with neurocognitive measures such as those outlined here? Do neurofeedback learning indices predict efficacy? At a theoretical level determination of the exact nature of the cognitive impairment is required, while at a methodological level there are numerous issues to be clarified such as training schedules, session length frequency and number, electrode placements and reward and inhibit bands. Furthermore, larger studies are warranted to examine whether the SMR protocol will be effective for the hyperactive subtype, and the beta1 protocol be effective for the inattentive subtype, and will both protocols be effective for the combined subtype? Questions such as, does one train on the basis of clinical diagnosis or EEG-based diagnosis need eventually to be addressed.

In conclusion, despite the validation work reported here much remains to be done to provide a scientific basis for biofeedback with the EEG spectrum. The monitoring of whole-scalp EEG and fMRI changes within each SMR, beta and al- 
pha-theta training session would also be of interest. Can our enhancement of attention and memory demonstrated in healthy subjects be extended to clinical groups? What are long-term influences on the EEG that accompany the cognitive improvements? Elucidation of the origin of the theta activity generated during alpha-theta training and the way in which the training may affect frontal beta band and metabolic activity is a high priority (Egner et al., 2004). The remarkable enhancement of artistic aspects of performance by alpha-theta training warrants application to the performing arts in general.

Of more immediate practical concern are the questions of who is most likely to benefit from the training and how to optimise the nature and duration of the training. These issues are at the present moment unresolved but under active investigation. Psychometric testing could possibly allow one to determine personality trait predictors of likely responsiveness to both SMR/beta and alpha/theta neurofeedback and successful performance enhancement (Hardman et al., 1997). The practical details of session length, schedule length, reward contingencies and electrode placements require controlled investigation.

Final, and crucial relevance of applying neurofeedback hinges decidedly on its potential to evoke long-term effects. The research to date does not permit any inferences regarding this important aspect with respect to alpha/theta training. All post-training music performance measures were taken within a time-span of maximally 4 weeks after the last training session. Regarding SMR and beta training in AD/DH children, there is suggestive evidence of long-term efficacy (Monastra et al., 2005). In the future, studies that involve regular follow-up assessments over a longer interval will have to be conducted in order to determine whether the costs of neurofeedback training both in terms of time and money as a clinical and performance enhancement tool are justified by long-term returns.

\section{Acknowledgment}

The research was undertaken with the support of the Leverhulme Trust, the Royal College of Music, Brain Health London and Cerebra the charity for the head injured child. The first author is currently in receipt of grants from the National Endowment for Science, Technology and Arts 
(NESTA) to extend the music results and the EU New Information Technologies, Creative Presence States to extend the work in the performing and originating arts.

\section{References}

Batty, M., Frick, A., Steffert, T., Hawken, M. and Gruzelier, J. (2005) A randomised control comparison of neurofeedback versus attention training in ADHD: a progress report. Meeting of the Society for Applied Neuroscience, Turkey, September, 2005.

Brown, B.B. (1970) Recognition of aspects of consciousness through association with EEG alpha activity represented by a light signal. Psychophysiology, 6: 442-452.

Budzynski, T. H. and Stoyva, J. M. (1972) Biofeedback techniques in behavior therapy. In: Shapiro D., Barber T.X., DiCara L.V., Kamiya J., Miller N.B. and Stoyva J.M. (Eds.), Biofeedback and Self-Control. Aldine, Chicago, pp. 437-459.

Clarke, A.R., Barry, R.J., McCarthy, R. and Selikowitz, M. (1998) EEG analysis in attentiondeficit/hyperactivity disorder: a comparative study of two subtypes. Psychiat. Res., 81: 19-29.

Clarke, A.R., Barry, R.J., McCarthy, R. and Selikowitz, M. (2001) Electroencephalogram differences in two subtypes of attention-deficit/hyperactivity disorder. Psychophysiology,

38: 212-221.

Donchin, E. and Coles, M.G.H. (1988) Is the P300 component a manifestation of context updating? Beh. Brain Sci., 11: 357-374.

Egner, T. and Gruzelier, J.H. (2001) Learned self-regulation of EEG frequency components affects attention and event-related brain potentials in humans. NeuroReport, 12: 4155-4159.

Egner, T. and Gruzelier, J.H. (2003) Ecological validity of neurofeedback: modulation of slow wave EEG enhances musical performance. NeuroReport, 14: 1221-1224.

Egner, T. and Gruzelier, J.H. (2004) EEG biofeedback of low beta band components: frequencyspecific effects on variables of attention and event-related brain potentials. Clin. Neurophysiol., 115: 131-139.

Egner, T., Strawson, E. and Gruzelier, J.H. (2002) EEG signature and phenomenology of alpha/theta neurofeedback training versus mock feedback. Appl. Psychophysiol. Biofeedback,, 27: 261-270.

Egner, T., Zech, T.F. and Gruzelier, J.H. (2004) The effects of neurofeedback training on the spectral topography of the healthy electroencephalogram. Clin. Neurophysiol., 115: 131-139.

Fuchs, T., Birbaumer, N., Lutzenberger, W., Gruzelier, J.H. and Kaiser, J. (2003) Neurofeedback treatment for attention-deficit/hyperactivity disorder in children: a comparison with methylphenidate. Appl. Pychophysiol. Biofeedback, 28: 1-12. 
Gomez, C.M., Vazquez, M., Vaquero, E., Lopez-Mendoza, D. and Cardoso, M.J. (1998) Frequency analysis of the EEG during spatial selective attention. Int. J. Neurosci., 95(1-2): 1732.

Green, D.M. and Swets, J.A. (1966) Signal Detection Theory and Psychophysics. Wiley, New York.

Gruzelier, J.H. (2006) EEG-neurofeedback and hypnosis: theta synchronization and PTSD. In: Roy, M. (Ed.), Novel Approaches to the Diagnosis and Treatment of Posttaumatic Stress Disorder. IOS Press, pp. 13-22.

Gruzelier, J.H. and Egner, T. (2004) Physiological self-regulation: biofeedback and neurofeedback. In: Williamon, A. (Ed.), Musical Excellence: Strategies and Techniques to Enhance Performance. Oxford University Press, pp. 197-219.

Gruzelier, J.H., Egner, T., Valentine, E. and Williamon, A. (2002) Comparing learned EEG selfregulation and the Alexander technique as a means of enhancing musical performance. In: Stevens, C., Burnham, D., McPherson, G., Schubert, E. and Renwick, J. (Eds.), Proceedings of the Seventh International Conference on Music Perception and Cognition Adelaide. Causal Productions, Sydney, Australia, pp. 89-92.

HansImayr, S., Sauseng, P., Doppelmayr, M., Schabus, M. and Klimesch, W. (2005) Increasing individual upper alpha power by neurofeedback improves cognitive performance in human subjects. Appl. Psychophysiol. Biofeedback,, 30: 1-10.

Hardman, E., Gruzelier, J., Cheesman, K., Jones, C., Liddiard, D., Schleichert, H. and Birbaumer, N. (1997) Frontal interhemispheric asymmetry: self regulation and individual differences in humans. Neurosci. Lett., 221: 117-120.

Hardt, J.V. and Kamiya, J. (1978) Anxiety change through electroencephalographic alpha feedback seen only in high alpha subjects. Science, 201: 79-81.

Kamiya, J. (1962) Conditioned Discrimination of the EEG Alpha Rhythm in Humans. Paper presented at the Western Psychological Association, San Francisco, CA.

Klimesch, W., Sauseng, P. and Gerloff, Ch. (2003) Enhancing cognitive performance with repetitive transcranial magnetic stimulation at human individual alpha frequency. Eur. J. Neurosci., 17: 1129-1133.

Kristeva-Feige, R., Fritsch, C., Timmer, J. and Lucking, C.H. (2002) Effects of attention and precision of exerted force on beta range EEG-EMG synchronization during a maintained motor contraction task. Clin. Neurophysiol., 113: 124-131.

Lantz, D. and Sterman, M.B. (1988) Neuropsychological assessment of subjects with uncontrolled epilepsy: effects of EEG biofeedback training. Epilepsia, 29: 163-171. 
Linden, M., Habib, T. and Radojevic, V. (1996) A controlled study of the effects of EEG biofeedback on cognition and behaviour of children with attention deficit disorder and learning disabilities. Biofeedback Self-Reg., 21: 35-51.

Lubar, J.O. and Lubar, J.F. (1984) Electroencephalographic biofeedback of SMR and beta for treatment of attention deficit disorders in a clinical setting. Biofeedback Self-Reg., 9: 1-23.

Lubar, J.F. and Shouse, M.N. (1976) EEG and behavioral changes in a hyperkinetic child concurrent with training of the sensorimotor rhythm (SMR): a preliminary report. Biofeedback Self-Reg., 1(3): 293-306.

Lubar, J.F., Swartwood, M.O., Swartwood, J.N. and O'Donnell, P.H. (1995) Evaluation of the effectiveness of EEG neurofeedback training for ADHD in a clinical setting as measured by changes in T.O.V.A. scores, behavioural ratings, and WISC-R performance. Biofeedback SelfReg., 20: 83-99.

Lynch, J.J., Paskewitz, D.A. and Orne, M.T. (1974) Some factors in the feedback control of human alpha rhythm. Psychosom. Med., 36: 399-410.

Monastra, V.J., Lubar, J.F. and Linden, M. (2001) The development of a quantitative electroencephalographic scanning process for attention deficit-hyperactivity disorder: reliability and validity studies. Neuropsychol., 15: 136-144.

Monastra, V.J., Lubar, J.F., Linden, M., VanDeusen, P., Green, G., Wing, W., Phillips, A. and Fenger, T.N. (1999) Assessing attention deficit hyperactivity disorder via quantitative electroencephalography: an initial validation study. Neuropsychol., 13: 424-433.

Monastra, V.J., Lynn, S., Linden, M., Lubar, J.F., Gruzelier,

J.H. and LeVaque, T.J. (2005) Electroencephalographic biofeedback in the treatment of attention-deficit/hyperactivity disorder. Appl. Psychophysiol. Biofeedback, 30: 95-114.

Peniston, E.G. and Kulkosky, P.J. (1989) Alpha-theta brainwave training and beta endorphin levels in alcoholics. Alcoholism Clin. Exp. Results, 13: 271-279.

Peniston, E.G. and Kulkosky, P.J. (1990) Alcoholic personality and alpha-theta brainwave training. Med. Psychother., 3: 37-55.

Peniston, E.G. and Kulkosky, P.J. (1991) Alpha-theta brainwave neurofeedback for Vietnam veterans with combat-related post-traumatic stress disorder. Med. Psychother., 4: 47-60.

Pfurtscheller, G. and Lopes da Silva, F.H. (1999) Event-related EEG/MEG synchronization and desynchronization: basic principles. Clin. Neurophysiol., 110: 1842-1857.

Plotkin, W.B. and Rice, K.M. (1981) Biofeedback as a placebo: anxiety reduction facilitated by training in either suppression or enhancement of alpha brainwaves. J. Consult Clin. Psychol., 49: 590-596. 
Pressner, J.A. and Savitsky, J.C. (1977) Effect of contingent and noncontingent feedback and subject expectancies on electroencephalogram biofeedback training. J. Consult Clin. Psychol., 45: 713-714.

Rasey, H.W., Lubar, J.F., McIntyre, A., Zoffuto, A.C. and Addott, P.L. (1996) EEG biofeedback for the enhancement of attentional processing in normal college students. J. Neurotherapy, 1(3): 15-21.

Raymond, J., Sajid, I., Parkinson, L.A. and Gruzelier, JH. (2005a) Biofeedback and dance performance: a preliminary investigation. Appl. Psychophysiol. Biofeedback, 30: 65-73.

Raymond, J., Varney, C. and Gruzelier, J.H. (2005b) The effects of alpha/theta neurofeedback on personality and mood. Cog. Brain Res., 23: 287-292.

Saxby, E. and Peniston, E.G. (1995) Alpha-theta brainwave neurofeedback training: an effective treatment for male and female alcoholics with depressive symptoms. J. Clin. Psychol., 51: 685693.

Scott, W.C., Kaiser, D., Othmer, S. and Sideroff, S.I. (2005) Effects of an EEG biofeedback protocol on a mixed substance abusing population. Am. J. Drug Alcolhol Abuse, 31: 455-469.

Shouse, M.N. and Lubar, J.F. (1979) Operant conditioning of EEG rhythms and ritalin in the treatment of hyperkinesis. Biofeedback Self-Reg., 4: 299-312.

Sterman, M.B. (1996) Physiological origins and functional correlates of EEG rhythmic activities: implications for self-regulation. Biofeedback Self-Reg., 21: 3-33.

Sterman, M.B. (2000) Basic concepts and clinical findings in the treatment of seizure disorders with EEG operant conditioning. Clin. Electroencephal., 31: 45-55.

Sterman, M.B. and Friar, L. (1972) Suppression of seizures in an epileptic following sensorimotor EEG feedback training. Electroencephal. Clin. Neurophysiol., 33: 89-95.

Sterman, M.B. and MacDonald, L.R. (1978) Effects of central cortical EEG feedback training on incidence of poorly controlled seizures. Epilepsia, 19: 207-222.

Sterman, M.B., MacDonald, L.R. and Stone, R.K. (1974) Biofeedback training of the sensorimotor electroencephalogram rhythm in man: effects on epilepsy. Epilepsia, 15: 395-416.

Sterman, M.B., Wyrwicka, W. and Roth, S.R. (1969) Electrophysiological correlates and neural substrates of alimentary behavior in the cat. Ann. NY. Acad. Sci., 157: 723-739.

Tansey, M.A. (1993) Ten-year stability of EEG biofeedback results for hyperactive boy who failed fourth grade perceptually impaired class. Biofeedback Self-Reg., 18(1): 33-44.

Tansey, M.A. and Bruner, R.L. (1983) EMG and EEG biofeedback training in the treatment of a 10-year-old hyperactive boy with a developmental reading disorder. Biofeedback Self-Reg., 8(1): 25-37. 
Thompson, L. and Thompson, M. (1998) Neurofeedback combined with training in metacognitive strategies: effectiveness in students with ADD. Appl. Psychophysiol. Biofeedback, 23(4): 243263.

Vazquez Marrufo, M., Vaquero, E., Cardoso, M.J. and Gomez,

C.M. (2001) Temporal evolution of alpha and beta bands during visual spatial attention. Brain Res. Cogn. Brain Res., 12(2): 315-320.

Vernon, D., Egner, T., Cooper, N., Compton, T., Neilands, C., Sheri, A. and Gruzelier, J.H. (2003) The effect of training distinct neurofeedback protocols on aspects of cognitive performance. Int. J. Psychophysiol., 47: 75-85.

Wyrwicka, W. and Sterman, M.B. (1968) Instrumental conditioning of sensorimotor cortex EEG spindles in the waking cat. Physiol. Behav., 3: 703-707. 